\title{
Estructura de las comunidades de peces de arrecife en las Islas Catalinas y Playa Ocotal, Pacífico Norte de Costa Rica
}

\author{
Mario Espinoza \& Eva Salas \\ Escuela de Biología, Universidad de Costa Rica, San Pedro M.O., San José 2060, Costa Rica. \\ marioespinozam@yahoo.com.mx; evamaria_cr@yahoo.com \\ Recibido 15-IV-2005. Corregido 01-VI-2005. Aceptado 10-VIII-2005.
}

\begin{abstract}
Structure of reef fish communities in Catalinas Islands and Ocotal beach, North Pacific of Costa Rica. The reefs are heterogeneous systems that maintain a high diversity of organisms. Fish community structure varies within and among reefs, so it would be expected that reef structure and heterogeneity should affect fish communities inhabiting reefs. Four reef patches at Catalinas Islands (Sur, La Pared, Roca Sucia and Sombrero)

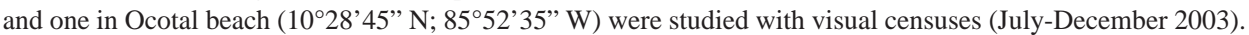
The structure and composition of fishes between Catalinas Islands and Ocotal beach were different, and habitat structure and composition explain most of the variance founded. The presence of the fleshy algae Caulerpa sertularioides in Ocotal, and the corals Tubastrea coccinea and Pocillopora spp. at Catalinas Island explained the variability among sites and how it affected fish community structure and composition. The butterfly fish Johnrandallia nigrirostris, damselfish Microspathodon dorsalis, and surgeon fish Prionurus punctatus were directly correlated with the ahermatipic coral Tubastrea coccinea in Roca Sucia reef, while the angel fish Holacanthus passer was associated to reefs with a major percentage of rocky substrate. Other species such as the damselfish Abudefduf troschelli and Halichoeres dispilus were more abundant at Ocotal, where the algae $C$. sertularioides dominated. The number and abundance of reef fishes was directly correlated with the rugosity index at the reefs of Roca Sucia and Ocotal, but not at reefs of La Pared and Sombrero. Rev. Biol. Trop. 53(3-4): 523-536. Epub 2005 Oct 3.
\end{abstract}

Key words: Community structure, substrate rugosity, coral reef fishes, habitat structure, diversity.

Los sistemas arrecifales son sitios que presentan una gran heterogeneidad estructural y en donde habitan complejas comunidades de peces de arrecife. Las diferencias en la composición de especies de un lugar a otro, inclusive en pequeñas áreas del arrecife, se pueden explicar por pequeñas diferencias en la estructura del sistema, que influyen en la colonización de diferentes especies adaptadas a sistemas similares (Ormond y Roberts 1997, Almany 2004).

La heterogeneidad espacial aumenta la diversidad de especies al proveer de refugios a las diferentes comunidades de peces de arrecife (Shulman 1984, Caley y St John 1996, Tilman 2001, Wolff 2002, Parra y Ruiz 2003). Recientemente, se le ha dado mucha importancia al tema de la riqueza de especies de peces y la complejidad del hábitat, lo que ha llevado a varios autores a estudiar la correlación entre la complejidad del hábitat con la diversidad de especies (Risk 1972, Luckhurst y Luckhurst 1978, Beukers y Jones 1997, Horan et al. 2000, Ferreira et al. 2001, Harding y Mann 2001, Gratwicke y Speight 2005). En los sistemas arrecifales, la mayoría de los peces son dependientes de un sustrato que les brinde protección así como alimento (Ryer y Olla 1995). Se esperaría, de este modo, que la heterogeneidad del sustrato vaya a influir en la estructura y composición de peces dentro del arrecife (Gratwicke y Speight 2005).

En varios estudios, se ha examinado la relación que existe entre algunas variables de 
los sistemas arrecifales, y la composición y estructura de las comunidades de peces de arrecife (Risk 1972, Gladfelter y Gladfelter 1978, Luckhurst y Luckhurst 1978, Carpenter et al. 1981, McManus et al. 1981, Aburto-Oropeza y Balart 2001, Bozec et al. 2005). Algunos de estos estudios han encontrado una correlación positiva entre la complejidad de la estructura del sustrato y la riqueza de especies (Risk 1972, Luckhurst y Luckhurst 1978, Carpenter et al. 1981), sin embargo otros autores no han encontrado ninguna relación (Sale y Dybdahahl 1975, Luckhurst y Luckhurst 1978, Roberts y Ormond 1987). Carpenter y colaboradores (1981) encontraron una fuerte correlación positiva en la complejidad del sustrato con la biomasa de peces, pero no así en la abundancia. Es por esto que es necesaria la utilización de métodos multivariados, en donde se pueden relacionar diferentes aspectos de la estructura y composición del hábitat con la estructura de las comunidades de peces de arrecife.

Este estudio relaciona la diversidad de las comunidades de peces de arrecife con el tipo de cobertura viva del sustrato, la rugosidad del sistema arrecifal y la composición del hábitat, en arrecifes rocosos de las Islas Catalinas y Playa Ocotal, Guanacaste. Este es el primer trabajo en describir las asociaciones de peces en estas localidades.

\section{MATERIALES Y MÉTODOS}

Descripción del sitio: Las Islas Catalinas están ubicadas en el Pacífico Norte de Costa Rica $\left(10^{\circ} 28^{\prime} 45^{\prime \prime} \mathrm{N}\right.$ y $\left.85^{\circ} 52^{\prime} 35^{\prime \prime} \mathrm{W}\right)$ a una distancia de aproximadamente $9 \mathrm{~km}$ de la costa. Constituyen un archipiélago de islas de origen volcánico, que forman importantes arrecifes rocosos, en los cuales se desarrollan varias comunidades coralinas que mantienen una alta diversidad de peces de arrecife. El arrecife de Ocotal, también ubicado en el Pacífico Norte (10³0'81' N y 8547'50' O), es un arrecife rocoso costero, dominado principalmente, por el alga Caulerpa sertularioides.
Muestreo: El estudio se realizó de julio a diciembre del 2003. Se escogieron cinco parches de arrecifes, cuatro de ellos ubicados en las Islas Catalinas y uno en Ocotal. Los diferentes sitios fueron escogidos debido al acceso, y por presentar diferencias en cuanto a la composición del hábitat, topografía y cobertura del sustrato. En cada sitio se estimó la composición de peces de arrecife. Para determinar la riqueza y abundancia de especies de peces de arrecife, se realizaron censos visuales mensuales en los arrecifes de La Pared, Catalinas Sur, Sombrero y Roca Sucia (I. Catalina) y en el arrecife de Ocotal. Sin embargo el arrecife de Catalina Sur no se utilizó en los análisis posteriores debido a la falta de mediciones. Para estimar la composición de especies, se utilizó el método de conteo puntual, anotando todas las especies de peces de arrecife dentro de un cilindro imaginario, durante un período de cinco minutos (Bohnsack y Bannerot 1986, Carlos y Samoilys 2000). El cilindro se marcó con una cuerda de nylon de $7.5 \mathrm{~m}$ de largo sobre el sustrato (radio del círculo).

La irregularidad y cobertura del sustrato se midieron en los arrecifes de La Pared, Sombrero y Roca Sucia (I. Catalinas) y en el arrecife de Ocotal. Se utilizó el índice de rugosidad (IR), para medir el porcentaje de rugosidad del sustrato (vario entre 0 y 1 ), dentro del mismo cilindro utilizado para el conteo de peces (Luckhurst y Luckhurst 1978). Se delimitó un transecto de $15 \mathrm{~m}$ de longitud (diámetro del círculo) y se colocó una cadena de $2.3 \mathrm{~m}$ de longitud con eslabones de $0.5 \mathrm{~cm}$ sobre el contorno del sustrato, en dos zonas distintas del transecto, para determinar la relación de la longitud real de la cadena y su longitud sobre el contorno del sustrato (IR). Para medir la cobertura se marcaron intervalos de 10 eslabones (15 cm de longitud en la cadena), y se estimó el porcentaje de cobertura en cada segmento (Luckhurst y Luckhurst 1978). La composición del hábitat se estimó visualmente en cada uno de los cilindros muestreados y se clasificó en: $\%$ sustrato rocoso, \% roca disgregada, \% arena, $\%$ corales, y $\%$ algas. 
Análisis estadístico: Para estimar la heterogeneidad y equidad de las comunidades de peces de arrecife se utilizó el índice de diversidad de Shannon-Wiener (H'), además se empleó el método de JackKnife para determinar la variabilidad asociada a H`. Se utilizó un Análisis Múltiple Discriminante (AMD) para determinar las variables que contribuían mayormente a la variabilidad respecto a la cobertura del sustrato, la composición visual del hábitat y la composición de especies (Digby y Kempton 1987). Además se realizó un Análisis de Correspondencia Canónica (ACC) para dilucidar relaciones entre las especies y variables del hábitat en los arrecifes del estudio. Los datos de cobertura del sustrato y composición del hábitat fueron transformados utilizando $\operatorname{arcseno} \sqrt{p}$, mientras que los datos de las especies se transformaron utilizando $\log _{10}(\mathrm{X}+1)$ (Krebs 1998).

\section{RESULTADOS}

Se observó un total de 2316 individuos, pertenecientes a 46 especies, distribuidas en 19 familias, siendo la damisela Chromis atrilobata la especie más abundante (Cuadro 1). La mayoría de las especies observadas pertenecen a las familias Pomacentridae, Labridae y Serranidae. Las damiselas de la familia Pomacentridae son principalmente herbívoros y se alimentan de algas y plancton, mientras que las señoritas de la familia Labridae y los meros y cabrillas de la familia Serranidae son importantes carnívoros, alimentándose de peces e invertebrados (Cuadro 1). En los arrecifes de La Pared y Ocotal se observó el mayor número de especies, sin embargo el número de individuos en ambos sitios fue diferente. La mayor diversidad se encontró en los arrecifes de Roca Sucia y Ocotal, respectivamente (Cuadro 2).

Al analizar la topografía del sustrato, los arrecifes de Roca Sucia y Sombrero presentaron los valores más altos de rugosidad (IR), además de la mayor diversidad de cobertura del sustrato (Cuadro 3). El IR varió entre 0.52 en el arrecife de Ocotal que era más plano, donde dominaba la arena, piedra disgregada y la $C$. sertularioides, hasta 0.71 , en zonas topográficas de mayor complejidad. Se encontró que en los arrecifes de Roca Sucia y Ocotal, la rugosidad del sustrato estaba correlacionada con el número de especies y la abundancia, pero no así en los arrecifes de La Pared y Sombrero (Cuadro 4).

Al estudiar los cambios en la composición de especies dentro de los arrecifes, el Análisis Múltiple Discriminante muestra que los arrecifes de Ocotal y Roca Sucia fueron muy diferentes al resto $\left(\lambda=2.82 ; \mathrm{F}_{36,9}=3.83\right.$, $\mathrm{p}=0.018$, Fig. 1). Los primeros 2 componentes explican un $96 \%$ de la variabilidad, que se debe principalmente, a que la composición de los peces observados en los arrecifes de Ocotal y Roca Sucia, fueron muy distintos al resto de los arrecifes, mientras que en los arrecifes de La Pared y Sombrero las comunidades de peces de arrecife fueron muy similares entre ellas (Fig. 1). En Roca Sucia se observó un mayor número de individuos de Prionurus punctatus, Johnrandalia nigrirostris, Holocanthus passer y Abudefduf troschelli, que fueron menos abundantes en el resto de los arrecifes (Cuadro 1).

En el Análisis Múltiple Discriminante, los primeros dos componentes explicaban el $96 \%$ de la variabilidad en la cobertura del sustrato $\left(\lambda=0.023 ; \mathrm{F}_{21,144}=18.487, \mathrm{p}<0.00001\right.$, Cuadro 5). La ausencia de rocas con algas filamentosas y los corales Pocillopora spp., además de la presencia de C. sertularioides fueron componentes importantes del sustrato en separar el arrecife de Ocotal del resto (Fig. 2, Cuadro 5). Los arrecifes de las I. Catalinas tienen un alto porcentaje de roca con algas filamentosas y de piedra disgregada (Fig. 3), mientras que en Ocotal domina el alga C. sertularioides en la cobertura del sustrato.

La composición del hábitat en los arrecifes de las I. Catalinas y Ocotal fue muy distinta. Se encontró una menor proporción de los corales del género Pocillopora y Porites, en Roca Sucia y La Pared, mientras que Tubastrea coccinea fue muy abundante en Roca Sucia. Estas diferencias fueron de gran importancia en separar los arrecifes (Fig. 4), en donde los primeros dos componentes del Análisis 
CUADRO 1

Lista de familias, de especies y número de peces de arrecife observados durante todo el estudio en los 5 arrecifes estudiados, Pacífico Norte de Costa Rica

Taxón

Acanthuridae

Prionurus punctatus

Acanthurus xanthopterus

Apogonidae

Apogon dovii

Balistidae

Aluterus scriptus

Pseudobalistes naugrafium

Sufflamen verres

Blennidae

Ophioblennius steindacneri

Plagiotremus asaleus

Chaetodontidae

Chaetodon humeralis

Johnrandalia nigrirostris

Cirrhithictidae

Cirrhitus rivulatus

Cirritichthys oxycephalus

Diodontidae

Diodon holocanthus

Diodon hystrix

Muraenidae

Gymnothorax castaneus

Muraena lentiginosa

Haemulidae

Haemulon flavigutatum

Haemulon maculicauda

Holocentridae

Sargocentrum suborbitalis

Kyphosidae

Kyphosus sp.

Labridae

Bodianus diplotaenia

Halichoeres chierchiae
Grupo

Trófico

Ocotal

Pare

Roca Sucia
Total

$\begin{array}{lllllll}\mathrm{A} & - & - & 37 & - & - & 37 \\ \mathrm{~A} & - & - & 3 & - & - & 3\end{array}$

1

I

I

I

A

$\mathrm{Pi}$

A/I/Pl

$\mathrm{A} / \mathrm{I} / \mathrm{Pl}$

$\mathrm{Pi} / \mathrm{C}$

Pi/C

C/M

C/M
$\mathrm{Pi} / \mathrm{C} / \mathrm{M}$

$\mathrm{Pi} / \mathrm{C} / \mathrm{M}$

I/P1

$\mathrm{I} / \mathrm{Pl}$

C

A

$\mathrm{Pi} / \mathrm{I}$

$\mathrm{Pi} / \mathrm{I}$
i/I

r

$\begin{array}{lllccc}8 & 9 & 10 & 14 & 10 & 51 \\ 3 & 1 & 11 & - & 5 & 20\end{array}$


CUADRO 1 (Continuación)

Lista de familias, de especies y número de peces de arrecife observados durante todo el estudio en los 5 arrecifes estudiados, Pacífico Norte de Costa Rica

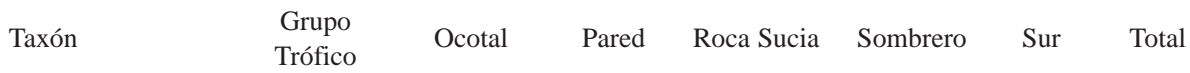

$\begin{array}{lcccccc}\text { Halichoeres dispilus } & \mathrm{Pi} / \mathrm{I} & 11 & 6 & 31 & - & 5 \\ \text { Halichoeres melanotis } & \mathrm{Pi} / \mathrm{I} & 1 & 6 & - & 1 & 2 \\ \text { Halichoeres nicholsi } & \mathrm{Pi} / \mathrm{I} & 4 & 6 & - & 16 & 83 \\ \text { Halichoeres notospilus } & \mathrm{Pi} / \mathrm{I} & - & 70 & - & 63 & - \\ \text { Thalassoma lucasanum } & \mathrm{Pi} / \mathrm{I} & 28 & 29 & 19 & 32 & 19\end{array}$

Lutjanidae

Lutjanus argentiventris

$\mathrm{Pi} / \mathrm{I}$

2

Pomacentridae

Abudefduf troschelli

Chromis atrilobata

Microspathodon dorsalis

Stegastes acapulcoensis

Stegastes flavilatus

Pomacanthidae

Holocanthus passer

Pomacanthus zonipectus

$\begin{array}{ccccccc}\text { A } & 19 & - & 26 & - & - & 45 \\ \text { Pl } & 188 & 390 & 155 & 145 & 291 & 1169 \\ \text { A } & 2 & - & 14 & - & - & 16 \\ \text { A } & 19 & - & 12 & - & 16 & 47 \\ \text { A } & 15 & 39 & 18 & 30 & 30 & 132\end{array}$

Scaridae

Scarus ghobban

Scarus rubroviolaceus

$\begin{array}{rrrrrrr}\mathrm{A} / \mathrm{C} / \mathrm{M} & 1 & 3 & 79 & 3 & 6 & 92 \\ \mathrm{~A} / \mathrm{C} / \mathrm{M} & 2 & 3 & - & - & - & 5\end{array}$

Serranidae

\section{Alphestes inmaculatus \\ Epinephelus labriformis \\ Epinephelus panamensis \\ Parantias colonus}

Serranus psittacinus

Tetraodontidae

Arothron hispidus
Arothron meleagris
Canthigaster punctatissima

Zanclidae

$$
\text { Zanclus cornutus }
$$

Total

Número de especies

$\begin{array}{lllllll}\mathrm{A} & - & 1 & 1 & - & - & 2 \\ \mathrm{~A} & - & 2 & - & - & - & 2\end{array}$

$\begin{array}{ccccccc}\mathrm{Pi} / \mathrm{C} & 1 & - & - & - & - & 1 \\ \mathrm{Pi} / \mathrm{C} & 1 & 4 & 4 & 7 & - & 16 \\ \mathrm{Pi} / \mathrm{C} & 1 & 13 & 4 & 5 & - & 23 \\ \mathrm{Pl} & - & 16 & 1 & - & - & 17 \\ \mathrm{Pi} / \mathrm{C} & 1 & - & - & 5 & - & 6\end{array}$

$\begin{array}{ccccccc}\mathrm{A} / \mathrm{I} & - & 3 & - & - & - & 3 \\ \mathrm{~A} / \mathrm{I} & 1 & 1 & - & 3 & - & 5 \\ \mathrm{~A} / \mathrm{I} & 6 & 1 & 8 & 1 & 2 & 18\end{array}$

$\mathrm{A} / \mathrm{I}$

$\begin{array}{cccccc}- & 4 & - & 4 & - & 8 \\ 352 & 635 & 579 & 341 & 409 & 2316 \\ 28 & 31 & 25 & 17 & 15 & 46\end{array}$

A - algas, I - invertebrados, Pi - piscívoro, Pl - plancton, $\mathrm{C}$ - crustáceos y $\mathrm{M}$ - moluscos. 


\section{CUADRO 2}

Estimación de la heterogeneidad y equidad según el índice de Shannon-Wiener, calculado con logaritmo en base 2, para las comunidades de peces de arrecife en 5 arrecifes del Pacífico Norte de Costa Rica

$\begin{array}{lccccc} & \text { Ocotal } & \text { Pared } & \text { Roca Sucia } & \text { Sombrero } & \text { Sur } \\ \mathrm{S} & 28 & 31 & 25 & 15 & 15 \\ \mathrm{H}^{\prime} & 2.83 \pm 1.02 & 2.38 \pm 0.33 & 3.43 \pm 0.42 & 2.52 \pm 0.18 & 1.81 \pm 0.64 \\ \mathrm{H}^{\prime} \max & 4.81 & 4.95 & 6.64 & 3.91 & 3.91 \\ \mathrm{~J}^{\prime} & 0.59 & 0.48 & 0.74 & 0.65 & 0.46\end{array}$

CUADRO 3

Valores del índice de rugosidad (I.R) y heterogeneidad y equidad, según el índice de Shannon-Wiener, calculado con logaritmo en base 2, para la cobertura del sustrato en 4 arrecifes del Pacífico Norte de Costa Rica

\begin{tabular}{|c|c|c|c|c|c|c|c|c|}
\hline & Ocotal & Pared & Roca Sucia & Sombrero & Arrecife & $\mathrm{H}^{\top}$ & Número especies & Abundancia \\
\hline I.R & 0.52 & 0.57 & 0.71 & 0.70 & Ocotal & 0.068 & $0.836^{* *}$ & $0.928^{* *}$ \\
\hline $\mathrm{H}^{\prime}$ & 1.38 & 2.15 & 1.69 & 2.15 & Pared & $0.60^{* *}$ & $-0.386^{* *}$ & $-0.222^{* *}$ \\
\hline \multirow[t]{2}{*}{ J } & 0.68 & 0.83 & 0.65 & 0.83 & Roca Sucia & $-0.662^{* *}$ & $0.805^{* *}$ & $0.561^{* *}$ \\
\hline & & & & & Sombrero & 0.014 & $-0.304^{* *}$ & $-0.724^{* *}$ \\
\hline
\end{tabular}

CUADRO 5

Funciones discriminantes de la cobertura del sustrato en 4 arrecifes del Pacífico Norte de Costa Rica (datos estandarizados entre variancias)

\begin{tabular}{lcc}
\multicolumn{1}{c}{ Sustrato } & $1^{\text {era }}$ Función Discriminante & $2^{\text {da }}$ Función Discriminante \\
Roca/algas filamentosas & 0.609 & 2.141 \\
Arena & -3.826 & 1.213 \\
Algas & 1.329 & -2.536 \\
Roca & 1.482 & 2.798 \\
Pocillopora sp. & 0.575 & 4.396 \\
Roca disgregada & -0.005 & 4.764 \\
Caulerpa sertularoides & -5.599 & 2.513
\end{tabular}




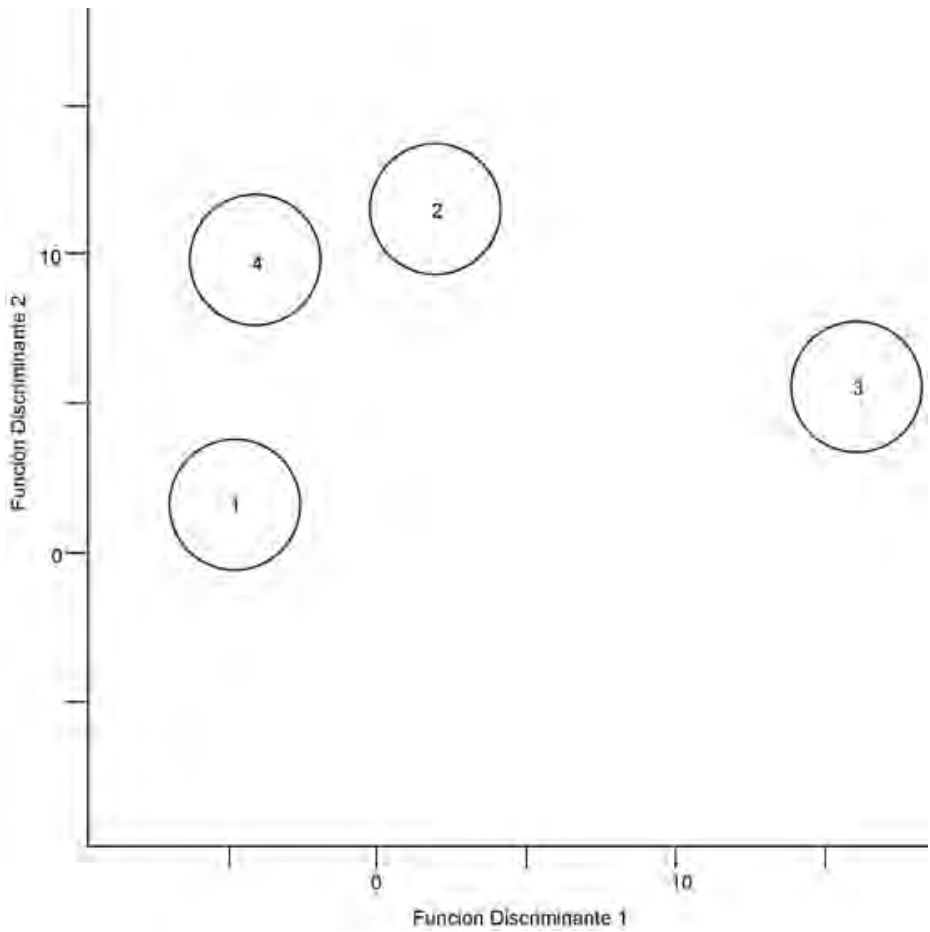

Fig. 1. Promedios de las funciones discriminantes que explican la variabilidad de la composición de especies en los 4 arrecifes estudiados (área de traslape al 95\% de confianza). Sitios: 1 - Ocotal, 2 - Pared, 3 - Roca Sucia, 4 - Sombrero.

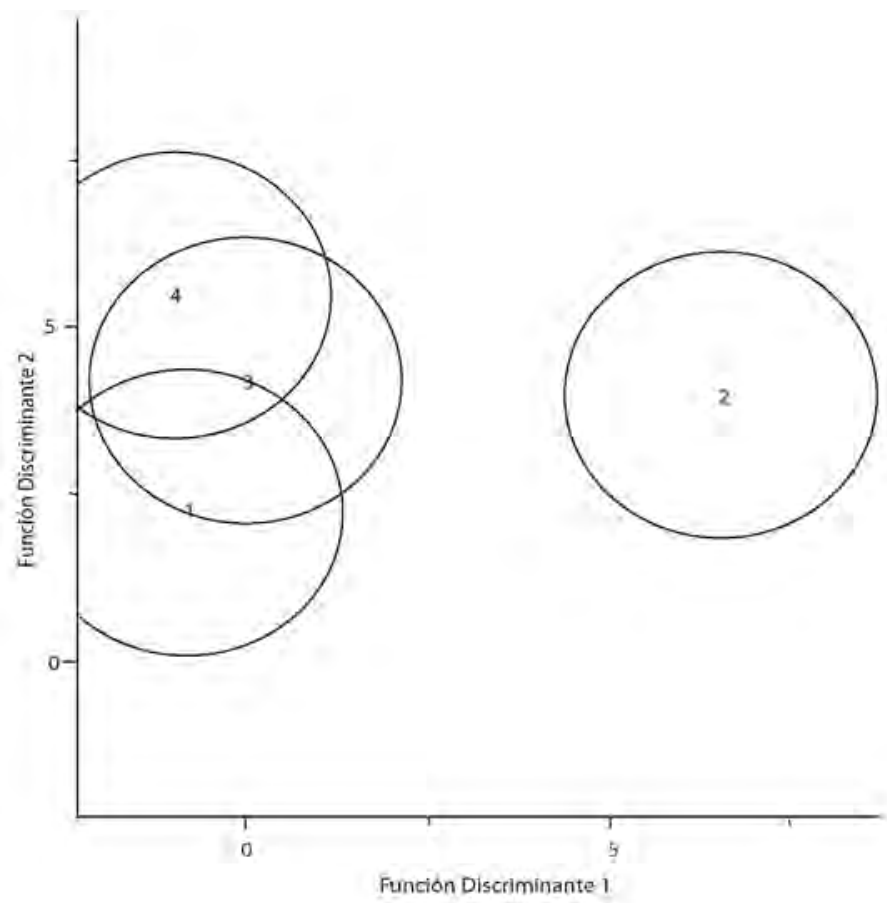

Fig. 2. Promedios de las funciones discriminantes que explican la variabilidad de la cobertura del sustrato en cada uno de los arrecifes estudiados (área de traslape al 95\% de confianza). Sitios: 1 - Ocotal, 2 - Pared, 3 - Roca Sucia, 4 - Sombrero. 


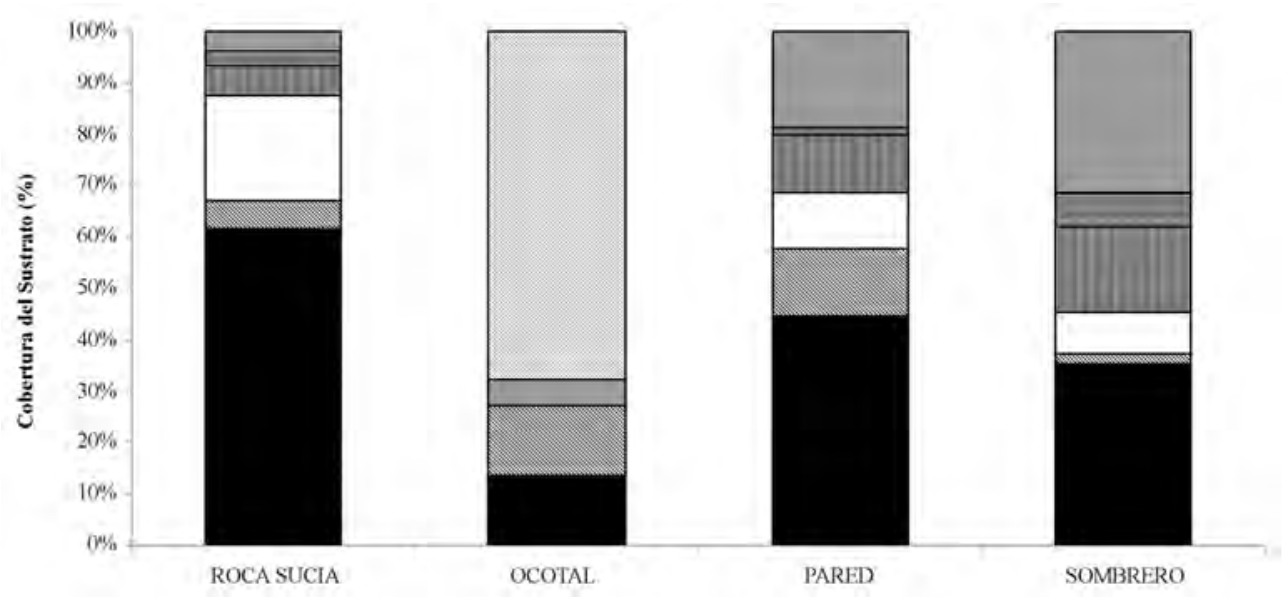

Fig. 3. Porcentaje de cobertura del sustrato de las variables de mayor importancia en 4 arrecifes, según el análisis múltiple discriminante. $\square$ Caulerpa sertularoides, $\square$ Roca disgregada, 目 Pocillopora, $\square$ Roca, $\square$ Algas, $\mathbb{\mathbb { N }}$ Arena, $\square$ Roca/algas filamentosas.

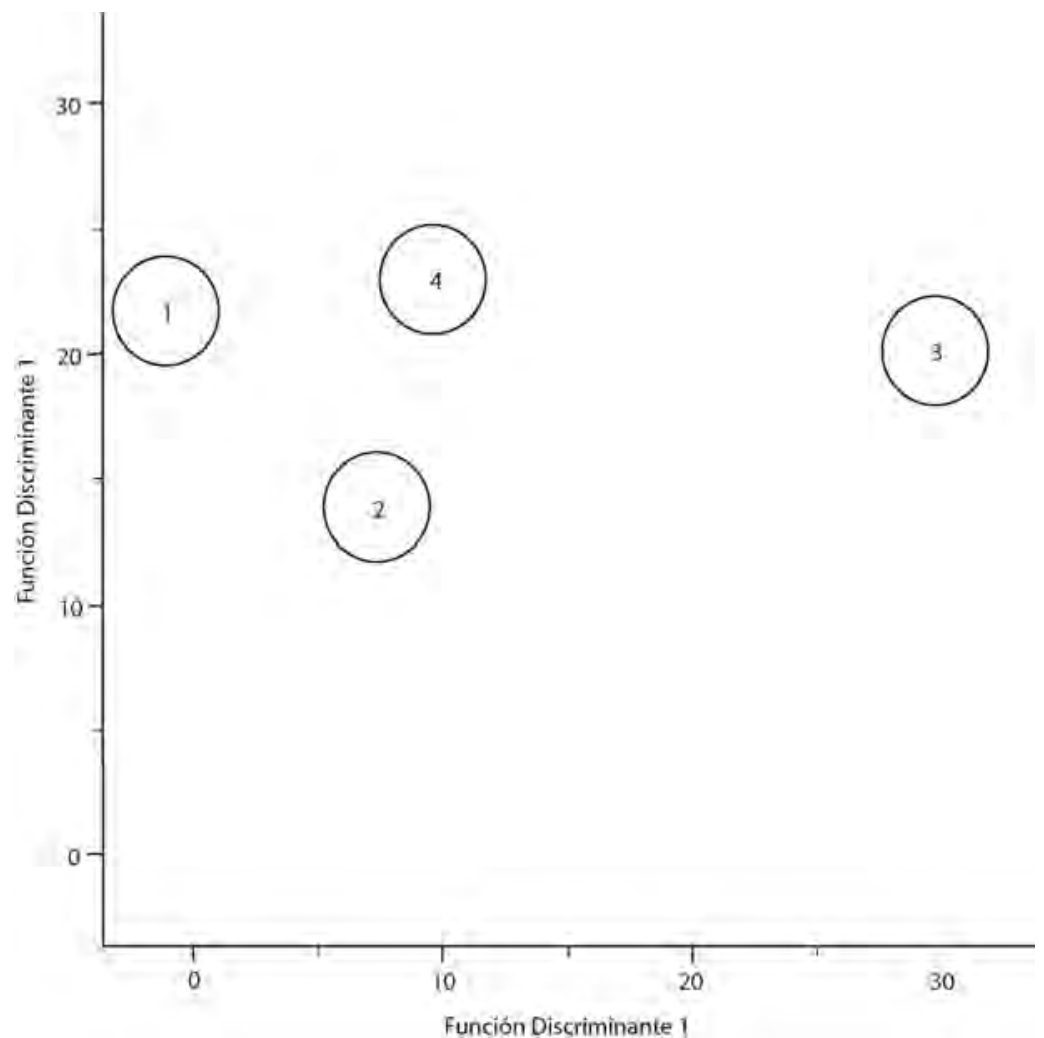

Fig. 4. Promedios de las funciones discriminantes que explican la variabilidad de la composición del hábitat en cada uno de los arrecifes estudiados (área de traslape al 95\% de confianza). Sitios: 1 - Ocotal, 2 - Pared, 3 - Roca Sucia, 4 - Sombrero. 
Múltiple Discriminante explicaban el $94 \%$ de la variabilidad del hábitat $\left(\lambda=0.0 ; \mathrm{F}_{24,15}=\right.$ 23.724, p < 0.0000, Cuadro 6). Además, el arrecife de Ocotal fue el único sitio en dónde se observó el alga C. sertularioides, dominando la cobertura del sustrato (Fig. 5).

En el Análisis de Correspondencia Canónica, se observó que varias de las especies más abundantes estaban fuertemente relacionadas con la estructura del hábitat (Fig. 6). Los primeros 2 ejes explicaban un $80 \%$ de la varianza (Cuadro 7). Las especies J. nigrirostris, Microspathodon dorsalis y $P$. punctatus estaban directamente correlacionados con el coral ahermatípico T. coccinea, presente únicamente en Roca Sucia, mientras que otras como A. troschelli y Halichoeres dispilus se correlacionan con el arrecife de Ocotal, en donde se encontraba el alga $C$. sertularioides.

\section{DISCUSIÓN}

La estructura y composición de las comunidades de peces de arrecife varió en abundancia y número de especies, entre y dentro de los arrecifes. Algunos estudios como el de Dominici (1996) y Tilman (2001) encontraron que varios

CUADRO 6

Funciones discriminantes de la composición del hábitat en 4 arrecifes del Pacífico Norte de Costa Rica (datos estandarizados entre variancias)

\begin{tabular}{lcc}
\multicolumn{1}{c}{ Hábitat } & $1^{\text {era }}$ Función Discriminante & $2^{\text {da }}$ Función Discriminante \\
Arena & 2.805 & 5.524 \\
Roca disgregada & 8.592 & 5.606 \\
Pocillopora sp. & -13.204 & 15.640 \\
Porites sp. & -16.053 & 27.858 \\
Tubastrea coccinea & -53.552 & 20.734 \\
Octocoral & -1.017 & -0.583 \\
Sustrato rocoso & -10.478 & 7.744 \\
Caulerpa sertularoides & 0.461 & 16.298
\end{tabular}

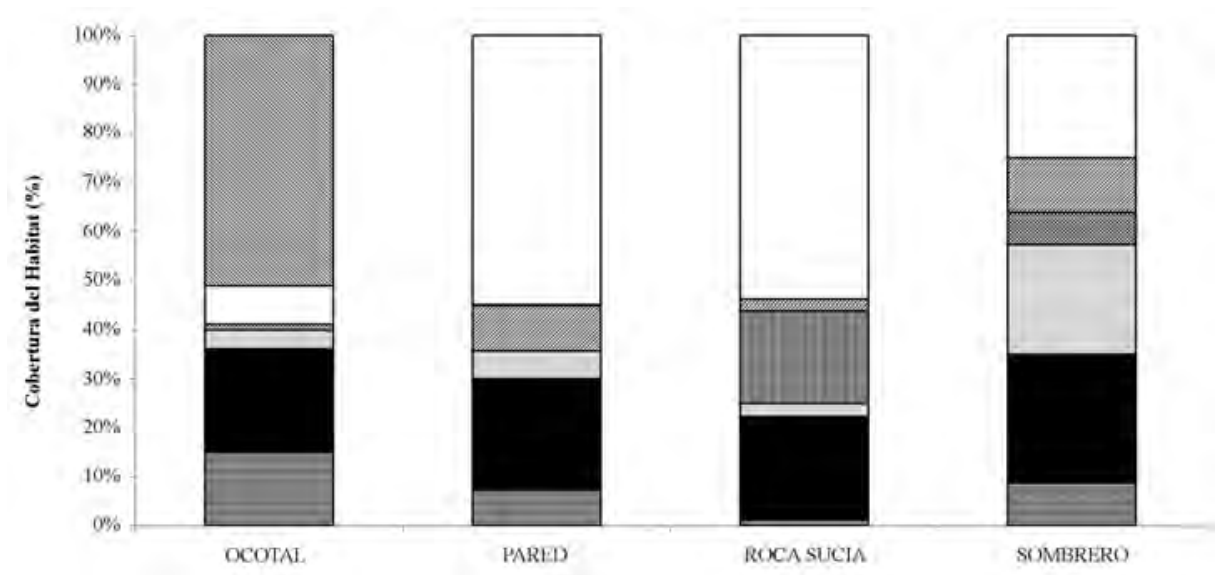

Fig. 5. Porcentaje de las variables de más importancia en la composición del hábitat en 4 arrecifes, según el análisis múltiple discriminante. $\mathbb{Q}$ Caulerpa sertularoides, $\square$ Sustrato rocosos, 国 Octocoral, $\square$ Tubastrea coccinea, 圆 Porites, $\square$ Pocillopora, $\square$ Roca disgregada, $⿴$ Arena. 
CUADRO 7

Porcentaje de la inercia y varianza explicada por las especies de peces de arrecife observados en los 5 arrecifes, en el Pacífico Norte de Costa Rica

\begin{tabular}{lcc}
\multicolumn{1}{c}{ Especie } & Inercia $(\%)$ & Varianza \\
Abudefduf troschelli & 15.27 & 1.48 \\
Bodianus diplotaenia & 1.9 & 0.21 \\
Chromis atrilobata & 7.64 & 0.28 \\
Halichoeres dispilus & 11.28 & 1.48 \\
Halichoeres nicholsi & 8.56 & 1.03 \\
Holacanthus passer & 4.58 & 0.44 \\
Johnrandalia nigrirostris & 11.61 & 1.25 \\
Microspathodon dorsalis & 9.57 & 1.51 \\
Prionurus punctatus & 13.7 & 2.2 \\
Stegastes flavilatus & 1.78 & 0.11 \\
Sufflamen verres & 1.31 & 0.37 \\
Thalassoma lucasanum & 2.27 & 0.14
\end{tabular}

sitios del Pacífico Norte de Costa Rica, presentan una alta diversidad de peces de arrecife que varían espacial y temporalmente. Estudios similares en el Pacífico Oriental (Dominici 1996, Zapata y Morales 1997) encontraron alrededor de 75 especies en 22 familias. Es posible que en I. Catalinas y Ocotal haya más especies, pero el tiempo y esfuerzo de nuestro estudio fue menor. Por ejemplo, buzos locales han informado a Manta birrostris, tiburones punta blanca Triaenodon obesus, cardúmenes de Rhinoptera steindachneri y Rhincodon typus como especies visitantes (Garrón, comm. pers), que no aparecieron en los censos.

La damisela planctívora Chromis atrilobata fue muy abundante en este estudio. En Bahía Culebra y en la Isla Gorgona los peces planctívoros dominan la estructura de las comunidades (Beukers y Jones 1997, Zapata y

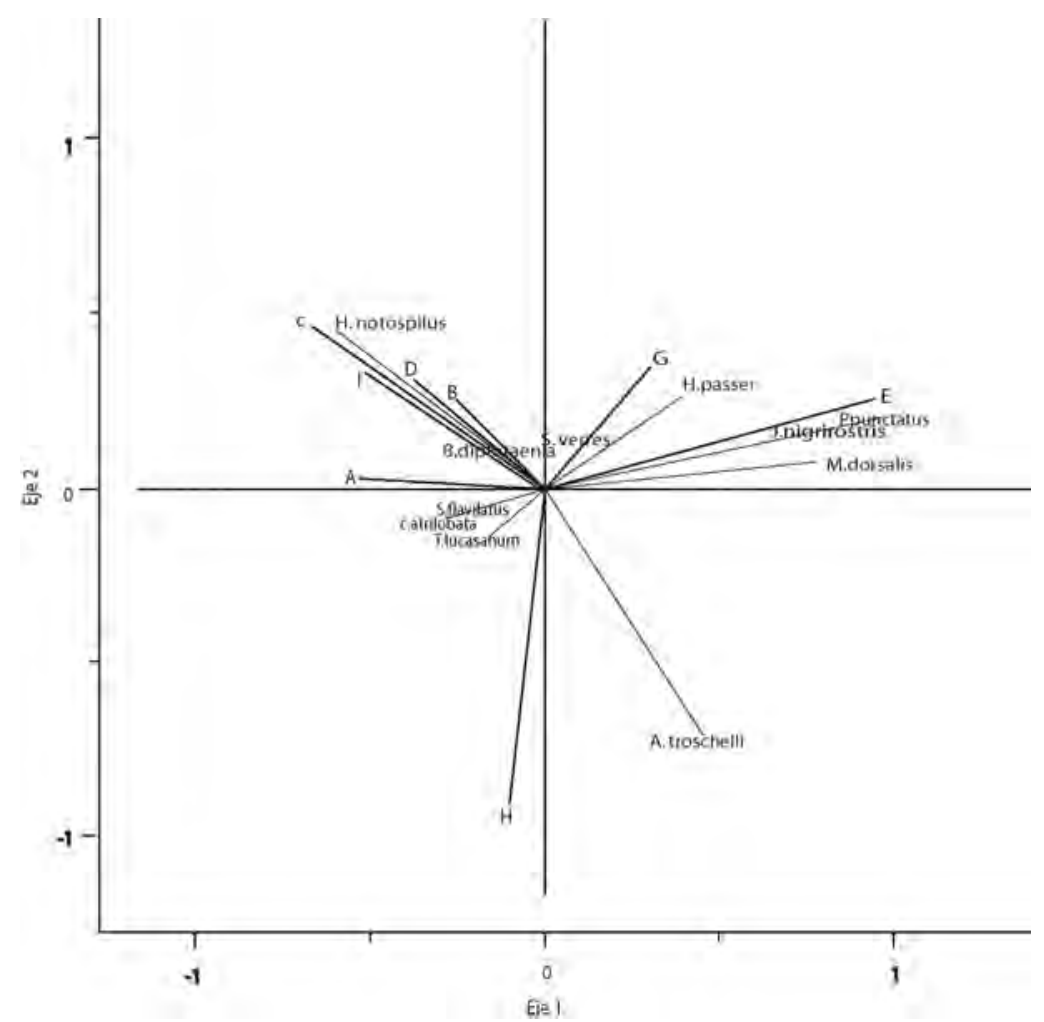

Fig. 6. Análisis de Correspondencia Canónica que muestra la relación entre las especies más importantes y las variables del hábitat en los distintos arrecifes del estudio. Composición del hábitat: A - arena, B - Roca disgregada, C - Pocillopora sp., D - Porites sp., E - Tubastrea coccinea, F - Octocoral, G - Roca, H - Caulerpa sertularoides. Eigenvalues: eje 1, 0.358; eje 2, 0.119. Los primeros 2 ejes $80 \%$ de la varianza. La prueba de Monte-Carlo en todos los ejes canónicos fue significativa $(\mathrm{p}<0.05), 1000$ permutaciones. 
Morales 1997, Dominici et al. 2003), lo que da apoyo a la hipótesis de que en Pacífico Oriental estos peces representan un alto componente de la biomasa, y posiblemente por diferencias interoceánicas en productividad marina. Sin embargo este patrón puede deberse al tipo de hábitat estudiado, que principalmente fue de pendientes rocosas, aparentemente este grupo predomina en pendientes arrecifales por la abundancia de su presa (Friedlander y Parrish 1998, Floeter et al. 2004).

Según los análisis de discriminante, las comunidades de peces de Roca Sucia y Ocotal fueron diferentes del resto, y la cobertura del sustrato explica la particularidad de Ocotal, mientras que la composición del hábitat destaca a Roca Sucia. Las escalas espaciales deben adecuarse a la pregunta y organismo de estudio (Sale 1998), y para investigar asociaciones de peces, nuestros resultados sugieren que deberían utilizarse ambas metodologías; la primera evalúa componentes del hábitat en una escala fina mientras que la segunda en una escala mayor.

En este estudio, se encontró que solo ciertas medidas de la estructura del hábitat explican diferencias entre arrecifes afectando la estructura de las comunidades de peces presentes. La topografía del sustrato parece tener una influencia directa en la diversidad y el índice de rugosidad puede ser un buen indicador de la heterogeneidad espacial de un sitio (Gratwicke y Speight 2005), lo cual podría explicar que el arrecife de Roca Sucia, con una mayor irregularidad del sustrato, tuviera un alto número de especies e individuos, sin embargo otros arrecifes como el de La Pared con un número alto de especies no presentaron una correlación directa.

Las diferencias de las comunidades de peces en Roca Sucia con respecto a los demás arrecifes, se cree que se deben a que en este arrecife, algunas especies fueron muy abundantes, entre ellas, J. nigrirostris y P. punctatus que se correlacionaban con el coral ahermatípico Tubastrea coccinea, presente únicamente en el arrecife de Roca Sucia. Una posible explicación se debe a que cuando A. troschelli se encuentra en etapa reproductiva protege agresivamente su masa de huevos dentro de su territorio (Robertson 1996), y se observó que J. nigrirostris, y P. punctatus, forman grandes cardúmenes para ingresar a los territorios de las damiselas y así alimentarse de las masas de huevos (Robertson et al. 1976, Freeman y Grossman 1992). Sin embargo, en Ocotal se encontró una alta abundancia de A. troschelli, y no así de estas especies que se alimentan de sus huevos. La presencia de buzos podría explicar la abundancia de $J$. nigrirostris y $P$. punctatus. Observaciones personales y de guías de buceo en la región han denotado que estas especies aprovechan la presencia del buzo en Roca Sucia para alimentarse de los huevos de la damisela, por lo que estas especies podrían estar en los otros arrecifes pero no ser tan conspicuas cuando entran los buzos por no tener este comportamiento aprendido. Sin embargo, el buceo se practica con regularidad en La pared y esto no ocurre. Otra hipótesis es que estas especies, principalmente $J$. nigrirostris son objeto de la pesca ornamental. La especie $H$. passer es también muy abundante en Roca Sucia y objeto de pesca ornamental (Dominici 1996). Este tipo de pesca se realiza con regularidad en Bahía Culebra, sitio donde se encuentra Ocotal. En las Islas Catalinas se da con menor frecuencia. Sin embargo estas hipótesis no terminan de explicar la particularidad de Roca Sucia respecto a los otros sitios. T. coccinea afecta la presencia de estas especies, y es necesario estudiar su alimentación.

La presencia de componentes del sustrato como coral Pocillopora sp., roca disgregada y la ausencia de Caulerpa sertularoides proporcionan gran parte de las diferencias observadas en los arrecifes de estudio, debido a que varias especies de peces de arrecife utilizan la heterogeneidad que proporcionan los corales para refugiarse. Tilman (2001) encontró que Thalassoma lucasanum muestra preferencias de acuerdo al tipo de cobertura de coral viva presente en el sustrato, en donde corales masivos del género Pavona aumentan la complejidad estructural del sistema, influenciando la preferencia de T. lucasanum, sobre corales ramificados del género Pocillopora. En los arrecifes de las Islas Catalinas y Ocotal 
la presencia del género Pavona fue muy baja o totalmente ausente, mientras que corales del género Pocillopora eran mucho más abundantes en los arrecifes de las Islas Catalinas. Aunque se esperaría que la cobertura de coral vivo tuviera una fuerte influencia en la diversidad de especies, este estudio no encontró ninguna relación directa, excepto para algunas especies y el coral T. coccinea (Fig. 8). Otros estudios (Sale 1984, Roberts y Ormond 1987, Reese 1997) también concluyen que la presencia de corales vivos en el sustrato no es suficiente para explicar la diversidad de peces de arrecife, y en el presente estudio solo se encontraron colonias aisladas que no formaban arrecifes. El alga $C$. sertularoides, domina la cobertura del sustrato en el arrecife de Ocotal y compite agresivamente con los corales, lo que podría estar afectando su crecimiento y determinando patrones de distribución espacial de varias especies (Neudecker 1979). Esta alga está invadiendo agresivamente los arrecifes aledaños (Fernández y Cortés 2005), y aún no se conoce su efecto en las comunidades de peces locales.

En conclusión, en los arrecifes de las Islas Catalinas y Ocotal varios atributos del arrecife fueron muy importantes entre y dentro de arrecifes. El dominio de C. sertularoides en Ocotal y su ausencia en el resto de los arrecifes estudiados tiene un efecto importante en la estructura de las comunidades que se encuentran en Ocotal. También el arrecife de Roca Sucia es el único sitio con un dominio del coral T. coccinea, siendo mucho más diferente a los demás arrecifes, en donde el comportamiento de forrajeo en grandes cardúmenes durante el periodo reproductivo de las damiselas afectó la presencia de mariposas, ángeles y cirujanos, encontrándose en mayor abundancia en este arrecife.

La topografía del sustrato se correlaciona con el número de especies y la abundancia de algunos arrecifes, siendo un buen estimador de la diversidad en el arrecife. Sin embargo, otros atributos del sistema pueden explicar mejor por qué ciertas especies se encuentran en un arrecife y no en otro, y cómo afecta esto a la estructura de la comunidad de peces.

\section{AGRADECIMIENTOS}

Agradecemos a Alejandro Solís y Marian Garrón de The EDGE Diving Company, por la gran colaboración en la parte logística durante los meses de muestreo. Además a Jeffrey Sibaja, por la colaboración en el análisis estadístico y a Jorge Cortés y Helena Molina por toda su ayuda durante el proyecto.

\section{RESUMEN}

Los arrecifes son sistemas muy complejos, cuya heterogeneidad mantiene una gran diversidad de organismos. La estructura de las comunidades de peces que forman parte del arrecife varía dentro y entre arrecifes, y se esperaría que la heterogeneidad y estructura del arrecife vaya a influir en la estructura de las comunidades de peces que habitan. Se estudiaron cuatro arrecifes en las Islas Catalinas (Sur, La Pared, Roca Sucia y Sombrero) y uno en la Playa de Ocotal, Pacífico Norte de Costa Rica. Se encontraron diferencias claras en la estructura y composición de las comunidades de peces de arrecife en las Islas Catalinas y Playa Ocotal, y la estructura y composición del hábitat explicaron la mayor parte de la varianza. Ciertos atributos del arrecife como la presencia del alga Caulerpa sertularoides en Ocotal, así como los corales Tubastrea coccinea y Pocillopora spp., en las Islas Catalinas explicaron la variabilidad que existía entre los sitios y como afectaba a la estructura de la comunidad de peces de arrecife. La mariposa Johnrandalia nigrirostris, la damisela Microspathodon dorsalis y el cirujano Prionurus punctatus se correlacionaron directamente con el coral ahermatípico Tubastrea coccinea, en el arrecife de Roca Sucia, mientras que Holocanthus passer se asocio a arrecifes con un mayor porcentaje de sustrato rocoso. Otras especies como Abudefduf troschelli y Halichoeres dispilus se encontraban en mayor cantidad en el arrecife de Ocotal, en donde dominaba el alga Caulerpa sertularioides. Por último, el número y abundancia de peces de arrecife se correlacionó directamente con el índice de rugosidad del sustrato en los arrecifes de Roca Sucia y Ocotal, pero no así los de la Pared y Sombrero.

Palabras clave: Estructura de comunidad, rugosidad del sustrato, peces de arrecife, estructura del hábitat, diversidad.

\section{REFERENCIAS}

Aburto-Oropeza, O. \& E. Balart. 2001. Community Structure of Reef Fish in Several Habitats of a Rocky Reef in the Gulf of California. Mar. Ecol. 22: 283-305. 
Almany, G.R. 2004. Does increased habitat complexity reduce predation and competition in coral reef fish assemblages? Oikos 106: 275-284

Bergenius, M.A. \& M.G. Meekan. 2002. Larval growth predicts the recruitment success of coral reef fish. Oecologia 131: 521-525.

Beukers, J.S. \& G.P. Jones. 1997. Habitat complexity modifies the impact of piscivores on a coral reef fish population. Oecologia 114: 50-59.

Bohnsack, J.A. \& S.P. Bannerot. 1986. A stationary visual census technique for quantitatively assessing community structure of coral reef fishes. NOAA Technical Report NMFS 41: 1-15.

Bosec, Y.M., S. Dolédec \& M. Kulbickit. 2005. An analysis of fish-habitat associations on disturbed coral reefs: chaetodontid fishes in New Caledonia. J. Fish. Biol. 66: 966-982.

Caley, M.J. \& J. St John. 1996. Refuge availability structures assemblages of tropical reef fishes. J. Anim. Ecol. 65: 414-428.

Carpenter, K.E., R.I. Miclat \& V.T. Corpus. 1981. The influence of substrate structure on the local abundance and diversity of Philippine reef fishes. Proc. $4^{\text {th }}$ Int. Coral Symp., Manila 2: 497-502.

Digby, P.G.N. \& R.A. Kempton. 1987. Multivariate Analysis of Ecological Communities. Chapman \& Hill, Londres. 543 p.

Dominici, A. 1996. Estructura poblacional de los peces de arrecifes del Golfo de Papagayo, Guanacaste, Costa Rica, con enfasis en las especies de mayor importancia commercial como ornamentales. Tesis de Maestria, Universidad de Costa Rica, San José, Costa Rica. 208 p.

Dominici, A., E. Brugnoli, J. Cortés, H. Molina \& M. Quezada. 2003. Community structure of eastern Pacific reef fishes (Gula of Papagayo, Costa Rica). Congreso Latinoaméricano Ciencias Marinas (colacmar). p. 291.

Fernández, C. \& J. Cortés. 2005. Caulerpa sertularioides, a green alga spreading aggressively over coral reef communities in Culebra Bay, North Pacific of Costa Rica. Coral Reefs. 24: 10.

Ferreira, C.E., J.E. Concalves \& R. Coutinho. 2001. Community structure of fishes and habitat complexity on a tropical rocky shore. Env. Biol. Fish. 61: 353-369.

Floeter, S.R., C.E Ferreira, A. Dominici-Arosemena \& I.R. Zalmon. 2004. Latitudinal gradients in Atlantic reef fish communities: trophic structure and spatial use patterns. J. Fish. Biol. 64: 1680-1699.

Freeman, M.C. \& G.D. Grossman. 1992. Group foraging by a stream minnow: shoal or aggregations? Anim. Behav. 44: 393-403.

Friedlander, A. M. \& J. D. Parrish. 1998. Habitat characteristics affecting fish assemblages on a Hawaiian coral reef. J. Exp. Mar. Biol. Ecol. 224: 1-30.

Gladfelter, W.B. \& E.H. Gladfelter. 1978. Fish community structures as a function of habitat structure on West Indian patch reefs. Rev. Biol. Trop. 26 (Suppl. 1): 65-84.

Gratwickle, B. \& M.R. Speight. 2005. The relationship between fish species richness, abundance and habitat complexity in a range of shallow tropical marine habitats. J. Fish. Biol. 66: 650-667.

Hardin, J.M. \& R. Mann. 2001. Oyster reefs as fish habitat: Opportunistic use of restored reefs by transient fishes. J. Shellfish Res. 20: 951-959.

Hixon, M.A. \& J.P. Beets. 1993. Predation, prey refuges, and the structure of coral-reef fish assemblages. Ecol. Monogr. 63: 77-101.

Honran, D.L., J.L Kershner, C.P Hawkings \& T.A. Crowl. 2000. Effects of habitat area and complexity on Colorado River cutthroat trout density in Uinta Mountain Stream. Trans. Am. Fish. Soc. 129: 12501263.

Luckhurst.B. \& K. Luckhurst. 1978. Analysis of the influence of substrate variable on coral reef fish communities. Mar. Biol. 49: 317-323.

McManus, J.W., R.I. Miclat \& V.P. Palaganas. 1981. Coral and fish community structure of Sombrero Island, Batangas, Philippines. Proc. Fourth Int. Coral Reef Symp., Manila 2: 271-280

Neudecker, S. 1979. Effects of grazing and browsing fishes on the zonation of corals in Guam. Ecology 60: 666-672.

Ormond, R.F.G. \& C.M. Roberts. 1997. Biodiversity of coral reef fish. Marine biodiversity: Patterns and Processes. Cambridge University, Cambridge, Inglaterra.

Parra, B. \& L.J. Ruiz. 2003. Estructura de la comunidad de peces en la costa oriental de la isla de cabagua, Venezuela. Rev. Biol. Trop. 51(Sup. 4): 197-203.

Reese, E.S. 1997. Predation on coral by fishes of the family Chaetodontidae: implications for conservation and 
management of coral reef ecosystems. Bull. Mar. Sci. 41: 594-604.

Risk, M.J. 1972. Fish diversity on a coral reef in the Virgin Islands. Atoll Res. Bull. 193: 1-6.

Roberts, C.M. \& R.F. Ormond. 1987. Habitat complexity and coral reef fish diversity and abundance on Red Sea fringing reefs. Mar. Ecol. Prog. Ser. 41: 1-8.

Ryer, C. \& B. Olla. 1995. Influences of food distribution on fish foraging behavior. Anim. Behav. 49: 411-418.

Robertson, D.R. 1976. Schooling as a mechanism for circumventing the territoriality of competitors. Ecology 57: $1208-1220$.

Robertson, D.R. 1996. Interspecific competition controls abundance and habitat use of territorial Caribbean damselfishes. Ecology 77: 885-899.

Sale, P.F. \& R. Dybdahl. 1975. Determinants of community structure for coral reef fishes in an experimental habitat. Ecology 56: 1343-1355.

Sale, P.F. 1978. Coexisting of coral reef fishes: a lottery for living space. Env. Biol. Fish. 3: 85-102.

Sale, P.F. 1984. Temporal variability in the community structure of fish on coral match reefs and the relation of community structure to reef structure. Ecology 65: 409-422.

Sale, P.F. 1998. Appropiate spatial scales for studies of reef-fish ecology. Aust. J.Ecol. 23: 202-208.

Samoilys, M.A. \& G.Carlos. 2000. Determining methods of underwater visual census for estimating the abundance of coral reef fishes. Env. Biol. Fish. 57: 289-304.

Shulman, M.J. 1984. Resource limitation and recruitment patterns in a coral reef fish assemblage. J. Exp. Mar. Biol. Ecol. 74: 85-109.

Tilman, J.A. 2001. The fisheries of Ornamental Fishes in Guanacaste, Costa Rica, with Special Emphasis on the Population Dynamics of the Cortez Rainbow Wrasse, Thalassoma lucasanum (Gill 1863). MSc Thesis in International Studies in Aquatic Tropical Ecology, Bremen, Alemania. 84 p.

Wolff, M. 2002. Concepts and approaches for marine ecosystem research with reference to the tropics. Rev. Biol. Trop. 50(2): 395-414.

Zapata, F.A. \& Morales, A. 1997. Spatial and temporal patterns of fish diversity in a coral reef at Gorgona Island, Colombia. Proc. $8^{\text {th }}$ Int. Coral Reef Symp., Panamá 1: 1029-1034. 